Canadian

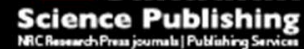

Canadian Journal of Microbiology Revue canadienne de de microbiologie

\title{
Effect of Tannic and Gallic Acids Alone or in Combination with Carbenicillin or Tetracycline on \\ Chromobacterium violaceum CV026 Growth, Motility, and Biofilm Formation
}

\begin{tabular}{|r|l|}
\hline Journal: & Canadian Journal of Microbiology \\
\hline Manuscript ID: & cjm-2015-0101.R1 \\
\hline Manuscript Type: & Article \\
\hline Date Submitted by the Author: & 30 -Apr-2015 \\
\hline Complete List of Authors: & $\begin{array}{l}\text { Dusane, Devendra; McGill University, Chemical Engineering } \\
\text { O'May, Che; McGill University, Chemical Engineering } \\
\text { Tufenkji, Nathalie; McGill University, Chemical Engineering }\end{array}$ \\
\hline \hline & antibiotic, biofilm, Chromobacterium violaceum, motility, tannins \\
\hline
\end{tabular}




\title{
1 Effect of Tannic and Gallic Acids Alone or in Combination
}

\author{
with Carbenicillin or Tetracycline on
}

3

4

5

6

7

8

9

15

16

17

18

11

3

4

15

17

8

\section{Biofilm Formation}

\section{Chromobacterium violaceum CV026 Growth, Motility, and}
Submitted to:
Canadian Journal of Microbiology
Devendra H. Dusane, Che O’May, Nathalie Tufenkji*

Department of Chemical Engineering, McGill University, Montreal, Quebec H3A 0C5,

Canada

Running title: Tannins modulate behaviour of $C$. violaceum

\footnotetext{
${ }^{*}$ Corresponding Author. Phone: (514) 398-2999; Fax: (514) 398-6678; E-mail: nathalie.tufenkji@mcgill.ca
} 
19 Abstract

20 Chromobacterium violaceum is an opportunistic pathogen which causes infections that are

21 difficult to treat. The goal of this research was to evaluate the effect of selected tannins (tannic

22 acid (TA) and gallic acid (GA)) on bacterial growth, motility, antibiotic (carbenicillin, 23 tetracycline) susceptibility, and biofilm formation. Both tannins, particularly TA, impaired

24 bacterial growth levels and swimming motilities at sub-MIC concentrations. In combination 25 with tannins, antibiotics showed increased minimum inhibitory concentration (MIC) values, 26 suggesting that tannins interfered with antibacterial activity. Sub-MIC concentrations of 27 tetracycline or TA alone enhanced biofilm formation of C. violaceum; however, in 28 combination, these compounds inhibited biofilm formation. In contrast, carbenicillin at sub29 MIC was effective in inhibiting $C$. violaceum biofilm formation; however, in combination 30 with lower concentrations of TA or GA, biofilms were enhanced. These results provide 31 insights into the effects of tannins on $C$. violaceum growth and their varying interaction with 32 antibiotics used to target $C$. violaceum infections.

36 Key words: antibiotic, biofilm, Chromobacterium violaceum, motility, tannins 


\section{Introduction}

39 Chromobacterium violaceum is a Gram-negative rod shaped bacterium found in soil and aquatic environments. This bacterium is not commonly studied; however, increasing number

41 of $C$. violaceum infections have been reported in recent years that warrant attention (Chen et

42 al. 2003; Alves de Brito et al. 2004; Yang and Li 2011). The bacterium is associated with 43 abscesses of the lung, liver, spleen, skin and can lead to fatal bacteremia (Sirinavin et al. 44 2005; Lim et al. 2009) and secondary infections such as diarrhea (Ballal et al. 2000; Chang et 45 al. 2007; Yang and Li 2011). These infections rapidly disseminate and due to failure of 46 antibiotic treatment the mortality rate is over 60\% (Yang and Li 2011). Multiple virulence factors affect bacterial pathogenesis including motility, biofilm 48 formation, and production of toxins (Bernard et al. 2012). C. violaceum cells can form 49 biofilms adhering to each other or to surfaces (Becker et al. 2009). Type III secretion, type IV 50 fimbriae, lipopolysaccharide synthesis, toxin and violacein production also influence 51 pathogenicity of C. violaceum (Alves de Brito et al. 2004). Beta-lactam antibiotics, cefoxitin, 52 ticarcillin, and tetracycline are used to treat C. violaceum infections (Farrar and Odell 1976; 53 Aldridge et al. 1988; Lee et al. 1999; Fantinatti-Garboggini 2004; Yang and Li 2011; Kumar 54 2012). However, recently, the bacterium showed resistance to $\beta$-lactam antibiotics (penicillin 55 and cephalosporin) due to acquired beta-lactamase and multidrug resistance genes (Martinez et al. 2000; Fantinatti-Garboggini et al. 2004). Due to the presence of multiple virulence

57 factors (motility, biofilm formation), multidrug resistance and scanty reports or guidelines on treatment methods, there is a growing interest in the study of alternative agents that may be 
Plant products containing tannins are promising candidates as alternatives to

61 antibiotics due to their antimicrobial properties (Haslam 1996; Cowan 1999; Serrano et al.

62 2009). Tannins are water-soluble polyphenols found in herbaceous and woody plants

63 (Schofield et al. 2001). Their antimicrobial activity may be due to (i) astringent property that

64 may induce complexation with enzymes or substrates, (ii) tannin's action on bacterial cell

65 membrane, or (iii) complexation of metal ions by tannins (Chung et al. 1998). Recent studies

66 show that tannins (e.g., tannic acid (TA), epigallogatechin gallate) can modify bacterial

67 virulence, motility and biofilm formation (Rasko and Sperandio 2010; O’May et al. 2012).

68 With increasing occurrence of antibiotic resistance and need for alternative chemical agents,

69 there is a necessity to understand the synergistic and antagonistic effects of tannins when used

70 in combination with common antibiotics. This information will provide insights into the

71 potential clinical applications and limitations of these phytochemicals (Chung et al. 1998).

72 Hence, this study examines the effects of the tannins TA and gallic acid (GA) on growth,

73 motility and biofilm formation by the less explored pathogen, C. violaceum.

74

75 Materials and methods

76 Bacterial strain and growth conditions

77 Chromobacterium violaceum strain CV026 (NCTC 13278) is a double-Tn5 mutant of the wild

78 type strain $C$. violaceum (ATCC 31532) obtained by mini-Tn5 transposon mutagenesis

79 (McClean et al. 1997). The transposon insertion sites have been mapped to a putative

80 repressor locus and to a luxI homologue (cviI) respectively. The wild type C. violaceum

81 (ATCC 31532) produces a violet color pigment, violacein, whereas the mutant C. violaceum

82 CV026 is unable to produce this pigment. We used the mutant strain (CV026) in this study 
83 because violacein interfered with the biofilm quantification assay. The bacterium was grown 84 in lysogeny broth (LB) (10 g/L tryptone, $5 \mathrm{~g} / \mathrm{L}$ yeast extract, and $5 \mathrm{~g} / \mathrm{L} \mathrm{NaCl})$ and on $\mathrm{LB}$ agar

85 (supplemented with $1 \%[\mathrm{w} / \mathrm{v}]$ agar). Pure stock cultures were maintained at $-80{ }^{\circ} \mathrm{C}$ in $30 \%$ $86(\mathrm{v} / \mathrm{v})$ frozen glycerol solutions in LB broth. Frozen cultures were streaked onto the LB agar 87 and incubated $\left(37^{\circ} \mathrm{C}, 24 \mathrm{~h}\right)$, and a colony of bacteria was inoculated into LB broth $(15 \mathrm{~mL})$ 88 and incubated $\left(37^{\circ} \mathrm{C}\right.$ at $\left.200 \mathrm{rpm}\right)$ for preparation of the bacterial inocula for the experiments.

\section{Chemical agents}

91 Tannic acid (TA) and gallic acid (GA) were purchased from Sigma-Aldrich (Canada). Stock 92 solutions $(5 \mathrm{mg} / \mathrm{mL})$ were prepared in deionized water (DI), the $\mathrm{pH}$ was adjusted to 7.0 (with $93 \mathrm{NaOH})$, filter sterilized $(0.1 \mu \mathrm{m}$ filters $)$ and stored at $4{ }^{\circ} \mathrm{C}$, protected from light. Antibiotics, 94 carbenicillin (CAR) and tetracycline (TET), were obtained from Sigma-Aldrich (Canada). 95 The antibiotics were dissolved in DI water, filter sterilized and stored at $4{ }^{\circ} \mathrm{C}$.

\section{MIC determination using standard agar well diffusion assay}

98 To determine the MIC of $\mathrm{TA}$ and GA on $C$. violaceum growth, the standard agar well 99 diffusion method was performed (Bennett et al. 1966). Briefly, exponentially grown cells of 100 C. violaceum were adjusted to $1 \times 10^{6}$ cells $/ \mathrm{mL}$ and an aliquot $(100 \mu \mathrm{L})$ was spread onto an $\mathrm{LB}$ 101 agar surface. Wells of diameter $6 \mathrm{~mm}$ were made in the agar plates and $50 \mu \mathrm{L}$ of tannins (15$1022000 \mu \mathrm{g} / \mathrm{mL}$ ) were added to the respective agar wells. Petri dishes were incubated at $37^{\circ} \mathrm{C}$ for $10324 \mathrm{~h}$ and inhibition zones were recorded. The experiments were performed in triplicate. The 104 MIC was defined as the lowest concentration of antibacterial agent that showed a zone of 105 inhibition $\geq 1 \mathrm{~mm}$ around the well. 


\section{MIC determination using microtitre growth assay}

108 Growth assays were performed in LB broth in non-tissue culture treated 96 well polystyrene 109 microplates (BD Falcon, USA). The culture was diluted (1:100) with LB broth in the presence 110 or absence of tannins and an aliquot $(200 \mu \mathrm{L})$ of this mixture was added to microtiter plate 111 wells. Medium without inoculum was used as controls. The plates were incubated at $37^{\circ} \mathrm{C}$ in 112 a microtiter plate reader (Tecan Infinite M200 Pro, Switzerland) and OD $_{600}$ was measured at 11330 min intervals for $24 \mathrm{~h}$ with shaking. The minimum inhibitory concentration (MIC) was 114 determined as the minimum concentration of TA or GA that showed no visible bacterial 115 growth in the wells (by $\left.\mathrm{OD}_{600}\right)$.

117 MIC determination using checkerboard assay of tannins and antibiotics against $C$. 118 violaceum

119 In the checkerboard assay, serial dilutions of antibiotics, CAR or TET, with tannins (TA or 120 GA) were mixed together in the microtiter plates so that each row or column contained a fixed 121 amount of one agent and increasing amount of the second. The concentrations of CAR ranged 122 from $0.04-50.0 \mathrm{mg} / \mathrm{mL}$. TET concentrations ranged from $0.4-500 \mu \mathrm{g} / \mathrm{mL}$. TA and GA 123 concentrations ranged from $0-2000 \mu \mathrm{g} / \mathrm{mL}$. The experiments were carried out in triplicate at $12437{ }^{\circ} \mathrm{C}$, for $24 \mathrm{~h}$ under shaking conditions of growth. After $24 \mathrm{~h}$, growth $\left(\mathrm{OD}_{600}\right)$ was 125 determined using the microtitre plate reader (Tecan Infinite M200 Pro). MIC was defined as 126 the minimum concentration tested that resulted in no visible bacterial growth in the wells 127 (clear well). 
129

130

131

132

133

134 135

136

137

138

139

140

141

142

143

144

145

146

147

148

149

150

\section{Effect of tannins and antibiotics on biofilm formation}

Biofilm formation was studied in flat bottom polystyrene 96 well microtiter plates (BD Falcon). The antibiotics (CAR and TET) and tannins (TA and GA) were tested for their ability to inhibit biofilm formation (for $24 \mathrm{~h}$ at $37^{\circ} \mathrm{C}$ ) in the microtiter plates. Antibiotics and tannins were used either alone or in combination at equivalent concentrations to those used in the checkerboard assay. C. violaceum CV026 was inoculated in $10 \mathrm{~mL}$ LB broth in $50 \mathrm{~mL}$ Falcon tubes (Ultident) and incubated at $37^{\circ} \mathrm{C}$ overnight at $150 \mathrm{rpm}$ shaking. The culture was diluted (1:100) with LB broth in the presence or absence of tannins and used for biofilm assays. Biofilms of $C$. violaceum were formed in non-tissue culture treated 96 well polystyrene microplates (BD Falcon, USA) for $24 \mathrm{~h}\left(37^{\circ} \mathrm{C}\right.$, static conditions) in the presence or absence of sub-MIC concentrations of tannins $(0-125 \mu \mathrm{g} / \mathrm{mL}$ in case of TA and $0-1000$ $\mu \mathrm{g} / \mathrm{mL}$ for GA) and antibiotics $(0-6 \mathrm{mg} / \mathrm{mL}$ in case of CAR and $0-7 \mu \mathrm{g} / \mathrm{mL}$ for TET). After incubation, the optical density $\left(\mathrm{OD}_{600}\right)$ of the culture was measured before removing the planktonic cells. Biofilms were analyzed by staining with crystal violet $(\mathrm{CV})$ as described elsewhere (O'Toole et al. 1999). Briefly, wells were gently washed twice with DI water to remove non-adherent, planktonic cells. After air drying $(10 \mathrm{~min}), 200 \mu \mathrm{L}$ of a $0.1 \% \mathrm{CV}$ solution was added to each well (15 min). Plates were rinsed with DI water, air dried and then $200 \mu \mathrm{L}$ of $95 \%$ ethanol was added to solubilize the CV stain bound to the surface-attached biofilms. The absorbance at $\mathrm{OD}_{595}$ (Tecan Infinite M200 Pro, Switzerland) was measured to estimate the biofilms that were formed. Biofilm levels $\left(\mathrm{OD}_{595}\right)$ were normalized to the level of bacterial growth $\left(\mathrm{OD}_{600}\right.$, determined separately) to decrease the potential bias in the measurements introduced due to differences in growth rate (Dunne 1990; Hosseinidoust et al. 
151 2013; Dusane et al. 2014). The biofilm levels reported represent the biofilm absorbance $152\left(\mathrm{OD}_{595}\right)$ normalized by the level of growth $\left(\mathrm{OD}_{600}\right)$.

153

154 Bacterial motility assays

155 Effect of tannins on bacterial motility was analyzed using swimming assays. Motility assays 156 were performed in polystyrene Petri dishes (diameter of $82 \mathrm{~mm}$ ) containing swim agar (LB 157 supplemented with $0.3 \%(\mathrm{w} / \mathrm{v})$ agar) and TA or GA at different concentrations. The plates 158 were dried for $1 \mathrm{~h}$ and inoculated with $5 \mu \mathrm{L}$ aliquots of mid-exponentially grown culture of $C$. 159 violaceum CV026. Inoculation was done within the agar (Tremblay et al. 2008) and the 160 diameters of the swimming motility zones were measured after incubation (for $20 \mathrm{~h}$ ) at $37^{\circ} \mathrm{C}$. 161 The experiments were carried out in triplicate.

162

163

Statistical analysis

164 Where indicated, a Student's $t$ - test was performed to compare the significance of the results, 165 with a $P$-value $<0.05$ considered as statistically significant.

166

167 Results

168 TA and GA growth effects on C. violaceum CV026

169 In the agar-diffusion method, TA and GA exhibited MIC values of 250 and $2000 \mu \mathrm{g} / \mathrm{mL}$, 170 respectively (Table 1$)$. When growth levels $(24 \mathrm{~h})$ were assessed in the microtiter plates, TA 171 exhibited a MIC of $500 \mu \mathrm{g} / \mathrm{mL}$, but concentrations as low as $7 \mu \mathrm{g} / \mathrm{mL}$ resulted in a decreased 172 growth rate. For GA, only the maximum concentration tested $(2000 \mu \mathrm{g} / \mathrm{mL})$ exhibited an 173 inhibitory effect (Fig. 1A and 1B). 
175 Combined effect of tannins and antibiotics on growth of $C$. violaceum (checkerboard 176 assay)

177 The MICs of the antibiotics (CAR) and (TET) were assessed alone and in combination with 178 TA and GA (Fig. 2A-D). In the checkerboard assay, TA and GA alone exhibited an MIC 179 value of 250 and $2000 \mu \mathrm{g} / \mathrm{mL}$, respectively. CAR alone exhibited an MIC of $12 \mathrm{mg} / \mathrm{mL}$, while 180 TET alone inhibited $C$. violaceum growth at a much lower MIC $(15 \mu \mathrm{g} / \mathrm{mL})$. The difference in 181 MIC values for TA in the agar-diffusion assay (Table 1) and the checkerboard assay may be 182 attributable to differences in the growth conditions (static versus shaking). When tannins and 183 antibiotics were combined, the MIC values of both antibiotics were increased (Fig. 2A-D). In 184 the presence of low concentrations of TA or GA, the MIC of CAR increased from 12 to 50 $185 \mathrm{mg} / \mathrm{mL}$ (Fig. 2A and 2B). For TET, the MIC increased from 15 to $30 \mu \mathrm{g} / \mathrm{mL}$ in TA and to $186125 \mu \mathrm{g} / \mathrm{mL}$ in case of GA. Low concentrations of antibiotics also enhanced the MIC for TA, 187 but not for GA at the concentrations tested. With CAR, the TA MIC increased from 250 to 188 $1000 \mu \mathrm{g} / \mathrm{mL}$, while TET $(\leq 3.5 \mu \mathrm{g} / \mathrm{mL})$ increased TA's MIC to $500 \mu \mathrm{g} / \mathrm{mL}$.

\section{Effect of tannins and antibiotics on biofilm formation by $C$. violaceum}

191 Tannins and antibiotics were tested alone and in combination to determine their effect on biofilm

192 formation in 96 well microtiter plates. Biofilm values $\left(\mathrm{OD}_{595}\right)$ were normalized by growth levels $193\left(\mathrm{OD}_{600}\right)$ to compensate for differences in the growth rate as described in the literature (Deighton 194 and Borland 1993; Pompilio et al. 2011; Hosseinidoust et al. 2013). Differences in biofilm 195 formation were observed in both normalized and non-normalized values, especially when tannins 196 were used in combination with the antibiotic TET (Figs. 3C, 3D and S1C, S1D). Only sub-MIC 197 concentrations of the antibiotics are included in Figs. 3 and S1. The MIC of CAR was previously 
198 shown to be $12 \mathrm{mg} / \mathrm{mL}$ (Fig. $2 \mathrm{~A}$ and $2 \mathrm{~B}$ ). With CAR $\geq 0.16 \mathrm{mg} / \mathrm{mL}$, reduction in the levels of 199 biofilm was observed as compared with controls without the antibiotic (Fig. 3A and 3B). At all 200 concentrations of $\mathrm{CAR} \leq 0.7 \mathrm{mg} / \mathrm{mL}$, addition of TA resulted in an enhancement of biofilm 201 formation (Fig. 3A). This occurred consistently with low TA (15 and $30 \mu \mathrm{g} / \mathrm{mL}$ ) concentrations, 202 but there were exceptions seen at the higher TA levels of 60 and $125 \mu \mathrm{g} / \mathrm{mL}$ (Fig. 3A). GA also 203 reduced the effectiveness of CAR to inhibit biofilm formation at certain concentrations (Fig. 3B). 204 However, the enhancement of biofilm by GA was less than with TA (Fig. 3A). Biofilm 205 formation was enhanced at CAR concentrations of 0.16 and $0.35 \mathrm{mg} / \mathrm{mL}$ and at 3 and $6 \mathrm{mg} / \mathrm{mL}$ 206 in combination with GA, especially at lower concentrations (30-125 $\mu \mathrm{g} / \mathrm{mL})$. The difference was 207 approximately two fold with $\mathrm{OD}_{595} / \mathrm{OD}_{600}$ ranging from 0.2 to 0.4 (Fig. 3B).

208 The MIC of TET was shown to be $15 \mu \mathrm{g} / \mathrm{mL}$ (Fig. 2C and 2D). With TET alone, 209 increase in the levels of biofilm was observed (Fig. 3C and 3D). When TA was added in the 210 absence of TET, biofilm formation was enhanced at concentrations $\leq 30 \mu \mathrm{g} / \mathrm{mL}$, but 211 decreased at TA concentrations $\geq 60 \mu \mathrm{g} / \mathrm{mL}$ (Fig. 3C). Interestingly, when TA was combined 212 with any given concentration of TET, there was a significant impairment of biofilm formation $213(P<0.05)$ compared to when no TA was present (Fig. 3C). This behavior was not generally 214 observed with the antibiotic CAR (Fig. 3A). GA was effective in inhibiting the biofilm 215 formation of $C$. violaceum when no antibiotic was present, and in the presence of CAR (at 216 higher concentrations of GA), whereas this inhibition did not occur in the presence of TET 217 (Fig. 3D). In summary, the combination of TA and TET was shown to be effective in 218 reducing the biofilms of $C$. violaceum. GA was not an effective anti-biofilm agent in 219 combination with the antibiotic TET. 


\section{Effect of tannin materials on motility}

222 Motility is important for the dissemination of pathogenic bacteria and the spread of infection 223 (McDougald et al. 2011; Rasko and Sperandio, 2010). Swimming motility was characterized 224 by migration of cells from the point of bacterial inoculation (Fig. 4A and 4B) in the swim 225 medium in the presence or absence of sub-MIC concentrations of tannins. C. violaceum 226 showed adequate swimming motility in the absence of tannins with swim zone diameters of $22740 \pm 6 \mathrm{~mm}$. In the presence of TA, swimming motility was significantly impaired at all 228 concentrations tested, with virtually no swimming at TA concentrations $\geq 30 \mu \mathrm{g} / \mathrm{mL}$ (Fig. 229 4A). GA also significantly impaired swimming motility at all concentrations tested, with 230 virtually no motility seen at GA concentrations $\geq 125 \mu \mathrm{g} / \mathrm{mL}$ (Fig. 4B).

232 Discussion

233 The study was undertaken to understand the synergistic and antagonistic effects of tannins 234 (TA and GA) in combination with antibiotics (CAR and TET) used in the treatment of $C$. 235 violaceum infections. Specifically, we investigated the effects of TA and GA (Fig. 5) against 236 C. violaceum with the goal of assessing their feasibility to be used as alternatives to 237 antibiotics or to enhance the effectiveness of existing antibiotics to mitigate bacterial growth 238 and biofilm formation. TA and GA impaired bacterial growth at MIC concentrations of 500 239 and $2000 \mu \mathrm{g} / \mathrm{mL}$, respectively (Fig. 1), demonstrating that TA has more antimicrobial activity 240 in comparison to that of GA (Table 1; Fig. 1). Similarly, Akiyama et al. (2001) reported that

241 TA exhibited greater antimicrobial activity in comparison to that of GA against different 242 strains of Staphylococcus aureus; with MIC values for TA of $250 \mu \mathrm{g} / \mathrm{mL}$ compared to MIC 243 values of $8000 \mu \mathrm{g} / \mathrm{mL}$ for GA. These reports demonstrate that TA has more antimicrobial 
244 activity in comparison to that of GA (Table 1; Fig. 1). One of the mechanisms of the effective 245 antibacterial activity of TA could be attributed to the presence of ester linkage between GA 246 and glucose (to form TA) (Chung et al. 1998) (Fig. 5). The inhibitory activity of GA at higher 247 concentrations could be due to the chelation of metal ions (cofactor) necessary for the 248 enzymatic activity and growth of bacterial cells (Liu et al. 2003; Yahiaoui et al. 2008). Chung 249 and Murdock (1991) reported the inhibitory effect of TA against Listeria monocytogenes, 250 Escherichia coli, Salmonella enteritidis, S. aureus, Aeromonas hydrophila and Streptococcus 251 faecalis. They also suggested that when TA is hydrolyzed, the ester bond is broken to form 252 gallic or ellagic acid resulting in the loss of antimicrobial property of TA. Also, the enhanced 253 antibacterial activity of TA, but not GA, could be due to the enhanced iron binding ability of 254 TA (Chung et al. 1998).

We tested the effectiveness of tannins (TA and GA) to influence the MIC of the 256 antibiotics, CAR and TET. These antibiotics were selected based on the reports of sensitivity 257 of C. violaceum towards the antibiotic CAR (Neu and Swarz 1969; Farrar and Odell 1976; 258 Yang and Li 2011) and TET (Lee et al. 1999; Kumar 2012). Of the 72 isolates of $C$. 259 violaceum tested against different antimicrobial agents, $42 \%$ were susceptible to CAR and 260 92\% to TET (Yang and Li 2011). C. violaceum has often been reported to develop resistance 261 to multiple antibiotics, including CAR and TET (Aldridge et al. 1988), so it can be useful to 262 identify adjunct therapies that enhance the activity of antimicrobials; therefore, the use of 263 tannins alone and in combination with selected antibiotics was tested. CAR is a carboxy264 penicillin antibiotic and TET is a broad spectrum polyketide antibiotic. In this study, the MIC 265 of CAR was $12 \mathrm{mg} / \mathrm{mL}$ and that of TET was $15 \mu \mathrm{g} / \mathrm{mL}$ (Fig. 2A-D). The resistance towards 266 CAR antibiotic is attributed to the ability of $C$. violaceum to produce beta-lactamase that 
267 breaks down the beta-lactam ring present in the penicillin group of antibiotics (Farrar and 268 Odell 1976; Fantinatti-Garboggini 2004). Reports also suggest the increased resistance of $C$. 269 violaceum over years of treatment towards the antibiotics CAR, cefoxitin and ticarcillin 270 (Aldridge et al. 1988). In the present investigation, when tannins were used in combination 271 with antibiotics, an increase in the MIC values of both antibiotics was observed (Fig. 2A-D).

272 We hypothesize that the reduction in antibacterial activity could be due to a) binding of the 273 antibiotic to tannins (Neyestani et al. 2007), b) competitive binding of the tannins to beta274 lactamase (in case of CAR) or similar enzymes responsible for antibiotic hydrolysis (Chung 275 and Murdock, 1991; Chung et al. 1998) or, c) tannins causing a modification of the bacterial 276 properties such as biofilm formation and/or secondary metabolite synthesis, rendering them 277 more protected to the effects of antibiotics (O'May et al. 2012). This study shows how different tannins affect the MIC of antibiotics against $C$. 279 violaceum. It is known that combination of antibiotics with natural agents can be an 280 interesting approach to control infections. As reported by Takahashi et al. (1995), an increase 281 in antibacterial activity against $S$. aureus was observed when antibiotics (oxacillin, 282 methicillin, aminobenzyl-penicillin, TET, and chloramphenicol) were used in presence of 283 tannins (catechin). This suggests that tannin containing compounds can enhance the 284 antibacterial activity of selected antibiotics. However, as per the results herein, this is not 285 always true. The MIC of CAR was increased from 12 to $50 \mathrm{mg} / \mathrm{mL}$ in presence of TA and 286 GA, and for TET there was an increase from 15 to $30 \mu \mathrm{g} / \mathrm{mL}$ with TA and 15 to $125 \mu \mathrm{g} / \mathrm{mL}$ 287 with GA (Fig. 2A-D). This has also been documented elsewhere, for example, green tea 288 extracts (containing tannins) reduced the anti-bacterial activity of amoxicillin and cephalexin 289 antibiotics against Streptococcus pyogens (Neyestani et al. 2007). Another study showed 
290 combination of TA and EGCG (epigallocatechin gallate) to enhance biofilm formation and 291 reduce antibacterial activity of tobramycin (O’May et al. 2012).

Bacterial motility is important for dissemination of the pathogen in the host, 293 acquisition of nutrients and in the establishment of bacterial virulence (McDougald et al.

294 2011). Plant materials containing tannins have been reported to restrict surface-associated 295 motility and, in turn, enhance biofilm formation in P. aeruginosa PAO1 (O'May et al. 2012). 296 The ability of the anti-motility compounds to enhance biofilm formation may be concerning 297 as the biofilm mode of growth is associated with enhanced resistance to antibiotics (Costerton 298 et al. 1999). Because C. violaceum is resistant to several antibiotics (Fantinatti-Garboggini 299 2004), we were interested in examining whether tannins are able to affect bacterial motility 300 and lead to the enhancement of biofilms. O'May et al. (2012) showed that the swarming 301 motility in P. aeruginosa PAO1 was effectively blocked by the application of TA, 302 epigallocatechin gallate, and cranberry powder; whereas, the formation of biofilms was 303 enhanced in the presence of these materials. In contrast, GA was less effective in blocking the 304 swimming motility and did not significantly affect the biofilms. To date, there is no evidence 305 in the literature that points to the inverse relationship between motility and the biofilm mode 306 of growth of $C$. violaceum. However, it would be expected that a reduction in bacterial 307 motility would correspond with an enhancement in the bacterial sessile lifestyle. In the 308 present study, TA significantly reduced swimming motility (Fig. 4A) and enhanced biofilm 309 formation of $C$. violaceum at lower concentrations (sub-MIC), but impaired biofilm formation 310 at $\mathrm{TA} \geq 60 \mu \mathrm{g} / \mathrm{mL}$ (Fig. $3 \mathrm{~A}$ and $3 \mathrm{C}$ ). Thus, the study herein provides support for an inverse 311 relationship when $C$. violaceum is exposed to sub-MIC concentrations of TA. However, there 312 was no increase in the biofilm levels at lower concentration of GA (in absence of antibiotic) 
313 and the inverse relationship (motility versus biofilm) was not observed in the presence of GA,

314 similar to the observations made by O’May et al. 2012. This study provides further support

315 that when identifying any anti-motility compound, care needs to be taken to understand

316 whether there will be any possible enhancement in biofilm formation or increased MIC of

317 already utilized antibiotics.

318 To evaluate an alternative strategy to combat $C$. violaceum infections, we tested the 319 susceptibility of this strain towards tannins (TA and GA) and antibiotics (CAR and TET). 320 Biofilm formation in 96 well plates was studied in the presence and absence of tannins and 321 antibiotics. TA at sub-MIC concentrations (15 and $30 \mu \mathrm{g} / \mathrm{mL})$ showed enhancement in the 322 formation of biofilms, but impaired biofilm at higher concentrations but lower than MIC (Fig. $3233 \mathrm{~A}$ and $3 \mathrm{C}$ ). On the other hand, addition of GA alone resulted in the reduction of biofilms at 324 sub-MIC concentrations 250-1000 $\mu \mathrm{g} / \mathrm{mL}$ (3B and 3D). The enhancement of biofilms in 325 presence of sub-MIC concentrations of tannins has been shown earlier by O'May et al. 326 (2012). The authors provided some evidence that interaction of TA with P. aeruginosa 327 flagella could be responsible for the enhancement of biofilms (O'May et al. 2012). The study 328 reported less biofilm formation of $P$. aeruginosa with flagellar mutants and tannins. They 329 hypothesized that tannins could cross-link the bacterial flagellum and result in inhibition of 330 swarming motility and enhanced biofilm formation. Similar interactions between the $C$. 331 violaceum flagellum and TA could have led to the increased biofilms in microtiter plates 332 observed herein. Tannin rich extracts of the plant Terminalia catappa have been reported to 333 inhibit biofilms of $P$. aeruginosa (Taganna et al. 2011). In a recent study, Trentin et al. (2013) 334 reported the ability of three plant extracts to prevent growth and inhibit the formation of $P$. 335 aeruginosa biofilms. They suggested the bacteriostatic effect of these tannin rich extracts to 
336 be responsible for the anti-biofilm effects. However, tannins may also interfere with the 337 activity of antibiotics. Herein, the antibiotic CAR alone was found to be effective in inhibiting 338 biofilms of C. violaceum at concentrations that did not prevent bacterial growth (Fig. 3A and 339 3B). However, in the presence of selected sub-MIC concentrations of TA or GA + antibiotics, 340 we observed enhanced biofilm formation (Fig. 3A and 3B). This shows that interaction of 341 tannins (especially TA) with CAR can result in antagonistic interactions, decreasing the 342 efficacy of the antibiotic (Fig. 2) and promoting biofilm formation (Fig. 3A and 3B). This 343 reduced efficacy of antibiotics could be due to binding of the tannins to antibiotics, thereby 344 reducing their bioavailability. However, when TA $(15-125 \mu \mathrm{g} / \mathrm{mL})$ was used in combination 345 with sub-MIC concentrations of TET, it effectively prevented formation of C. violaceum 346 biofilms (Fig. 3C). This result is interesting because bacterial growth was not inhibited (Fig. 347 2C), and bacteria were not forming biofilms either. Similarly, GA in combination with CAR 348 at selected concentrations (0.08 and $1.5 \mathrm{mg} / \mathrm{mL} \mathrm{CAR})$ showed reduction in biofilms (Fig. 3B). 349 The reduction in biofilms was however to a lower extent as compared with TA + TET. This 350 suggests that TA can work synergistically with TET to prevent biofilm development. The 351 synergistic relationship was not observed with TA and CAR. TET antibiotic inhibits bacterial 352 protein synthesis by binding to the 30S ribosomal subunit (Chopra and Roberts 2001) and 353 CAR is a beta-lactam antibiotic that inhibits bacterial cell wall synthesis (Butler et al. 1970). 354 TA has a greater relative binding efficiency to iron than GA, wherein TA acts like 355 siderophores and chelates iron from the medium making it unavailable to the microorganisms 356 for growth and virulence. This could be one of the mechanisms of increased efficacy of TA 357 (Chung et al. 1998) compared to GA. It is also worth noting that biofilm levels appear to be 358 enhanced when the data is not normalized by the level of bacterial growth $\left(\mathrm{OD}_{600}\right)$ (Fig. S1). 
359 These results illustrate the importance of considering the normalized extent of biofilm 360 formation which takes into account the growth inhibitory effects of TA, GA or antibiotics 361 (Fig. 3). The study also suggests that the combined application of TA with TET or GA with 362 CAR (at selected concentrations) for preventing the formation of bacterial biofilms might be a 363 useful treatment approach. However, caution must be applied to avoid the development of 364 enhanced bacterial resistance. This study showed that tannins could have either positive or negative effects towards 366 the bacterium, C. violaceum. Overall, tannins were effective in modulating the behaviour of 367 the relatively less explored pathogen, C. violaceum. TA was more effective in controlling 368 bacterial growth and motility as compared to GA. At sub-MIC concentrations $(15-30 \mu \mathrm{g} / \mathrm{mL})$, 369 TA enhanced biofilm formation; however, in the presence of the antibiotic TET (0.4-7 $370 \mu \mathrm{g} / \mathrm{mL}$ ), it inhibited biofilms of $C$. violaceum. Further study with tannins in combination with 371 different antibiotics and using different biofilm models is needed to obtain an improved 372 understanding of the underlying mechanisms of action for such alternative strategies that 373 could be made available to control C. violaceum infections.

375 Acknowledgements The authors acknowledge the financial support of the Natural Sciences 376 and Engineering Research Council of Canada (NSERC) and the Canada Research Chair 377 (CRC) Program. They thank RJC McLean (Texas State University) for providing the $C$. 378 violaceum CV026 strain.

\section{References}


381 Akiyama, H., Fujii, K., Yamasaki, O., Oono, T., and Iwatsuki, K. 2001. Antibacterial action 382 of several tannins against Staphylococcus aureus. J. Antimicrob. Chemother. 48(4): 487-491 383 doi:10.1093/jac/48.4.487

384 Aldridge, K.E., Valaninis, G.T., and Saners, C.V. 1988. Comparison of the in vitro activity of 385 ciprofloxacin and 24 other antimicrobial agents against clinical strains of Chromobacterium 386 violaceum. Diag. Microbiol. Infect. Dis. 10: 31 e9

387 Alves de Brito, C.F., Carvalho, C.M., Santos, F.R., Gazinell, R.T., Oliveira, S.C., Azevedo, 388 V., et al. 2004. Chromobacterium violaceum genome: molecular mechanisms associated with 389 pathogenicity. Genetic Mol. Res. 3: 148-161.

390 Ballal, M., Kini, P., Rajeshwari, D., and Shivananda, P.G. 2000. Chromobacterium violaceum 391 diarrhea. Indian J. Pediatr. 67: 388-389.

392 Becker, S., Soares, C., and Porto L.M. 2009. Computational analysis suggests that virulence 393 of Chromobacterium violaceum might be linked to biofilm formation and poly-NAG 394 biosynthesis. Genet. Mol. Biol. 32: 640-644.

395 Bennett, J.V., Brodie, J.L., Benner, E.J., and Kirby, W.M.M. 1966. Simplified, accurate 396 method for antibiotic assay of clinical specimens. Appl. Microbiol. 14: 170-177.

397 Bernard, C.S., Giraud, C., Spagnolo, J., de Bentzmann, S. 2012. Biofilms: The secret story of 398 microbial communities. In Locht, C., and Simonet, M. (eds). Bacterial pathogenesis: 399 Molecular and cellular mechanisms. Horizon Scientific Press 129-168.

400 Butler, K., English, A.R., Ray, V.A., Timreck, A.E. 1970. Carbenicillin: chemistry and mode of 401 action. J. Infect. Dis. 122: S1-8. 
402 Chang, C.Y., Lee, Y.T., Liu, K.S., Wang, Y.L., and Tsao, S.M. 2007. Chromobacterium 403 violaceum infection in Taiwan: a case report and literature review. J. Microbiol. Immunol. $404 \quad$ Infect. 40: 272-275.

405 Chen, C.H., Lin, L.C., Liu, C.E., and Young, T.Z. 2003. Chromobacterium violaceum 406 bacteremia: a case report. J. Microbiol. Immunol. Infect. 36: 141-144.

407 Chopra, I., Roberts, M. 2001. Tetracycline antibiotics: mode of action, applications, molecular 408 biology, and epidemiology of bacterial resistance. Microbiol.Mol. Biol. Rev. 65(2): 232-260. 409 doi:10.1128/MMBR.65.2.232-260.2001.

410 Chung, K.T., Wong, T.Y., Huang, Y.W., and Lin, Y. 1998. Tannins and human health: a 411 review. Crit. Rev. Food. Sci. Nutr. 38: 421-464.

412 Chung, K.T., and Murdock, C.A. 1991. Natural systems for preventing contamination and 413 growth of microorganisms in foods. Food Structure 10: 361-374.

414 Costerton, J.W., Stewart, P.S., and Greenberg, E.P. 1999. Bacterial biofilms: a common cause 415 of persistent infections. Science 284(5418): 1318-1322.

416 Cowan, M.M. 1999. Plant products as antimicrobial agents. Clin. Microbiol. Rev. 12: 564417582.

418 Deighton, M., and Borland, R. 1993. Regulation of slime production in Staphylococcus 419 epidermidis by iron limitation. Infect. Immun. 61: 4473-4479.

420 Dunne, W.M. 1990. Effects of subinhibitory concentrations of vancomycin or cefamandole on 421 biofilm production by coagulase-negative staphylococci. Antimicrob. Agents Chemother. 422 34(3): 390-393. doi: 10.1128/aac.34.3.390 
423 Dusane, D. H., Hosseinidoust, Z., Asadishad, B., and Tufenkji, N. 2014. Alkaloids modulate 424 motility, biofilm formation and antibiotic susceptibility of uropathogenic Escherichia 425 coli. PLoS ONE 9(11): e112093. doi: 10.1371/journal.pone.0112093

426 Fantinatti-Garboggini, F., Almeida, R., Portillo, V.A., Barbosa, T.A.P., Trevilato, P.B., Neto, 427 C.E.R., et al. 2004. Drug resistance in Chromobacterium violaceum. Genet. Mol. Res. 3: 134428147.

429 Farrar, W.E., Jr., and Odell, N.M. 1976. Beta lactamase activity in Chromobacterium 430 violaceum. J. Infect. Dis. 134: 290-293.

431 Haslam, E. 1996. Natural polyphenols (vegetable tannins) as drugs: possible modes of action.

432 J. Nat. Prod. 59(2): 205-215.

433 Hosseinidoust, Z., Tufenkji, N., van de Ven, T.G.M. 2013. Formation of biofilms under phage 434 predation: considerations concerning a biofilm increase. Biofouling 29: 457-468.

435 Kumar, M.R. 2012. Chromobacterium violaceum: A rare bacterium isolated from a wound 436 over the scalp. Int. J. Appl. Basic Med. Res. 2(1): 70-72.

437 Lim, I.W., Stride, P.J., Horvath, R.L., Hamilton-Craig, C.R., and Chau, P.P. 2009. 438 Chromobacterium violaceum endocarditis and hepatic abscesses treated successfully with 439 meropenem and ciprofloxacin. Med. J. Austr. 190: 386-387.

440 Liu, I.C., Hsu, F.L., Tsai, T.C., Chan, P., Liu, J.Y.H., Thomas, G.N., et al. 2003. 441 Antihypertensive effects of tannins isolated from traditional Chinese herbs as non-specific 442 inhibitors of angiotensin converting enzyme. J. Bacteriol. 73: 1543-1555.

443 Martinez, R., Velludo, M.A., Dos Santos, V.R., and Dinamarci, P.V. 2000. Chromobacterium 444 violaceum infection in Brazil. A case report. Revista do Instituto de Medicina Tropical de Sao 445 Paulo 42: 111-113. 
446 McClean, K.H., Winson, M.K., Fish, L., Taylor, A., Chhabra, S.R., Camara, M., et al. 1997. 447 Quorum sensing and Chromobacterium violaceum: exploitation of violacein production and 448 inhibition for the detection of N-acylhomoserine lactones. Microbiol. 143: 3703 - 3711.

449 McDougald, D., Rice, S.A., Barraud, N., Steinberg, P.D., and Kjelleberg, S. 2011. Should we 450 stay or should we go: mechanisms and ecological consequences for biofilm dispersal. Nature 451 Rev. Microbiol. 10(1): 39-50.

452 Neu, H.C., and Swart, H. 1969. Resistance of Escherichia coli and Salmonella typhimurium to 453 carbenicillin. J. Gen. Microbiol. 58: 301-305.

454 Neyestani, T.R., Khalaji, N., and Gharavi, A. 2007. Selective microbiological effects of tea 455 extract on certain antibiotics against Escherichia coli in vitro. J. Alt. Complement. Med. 456 13(10): 1119-1124.

457 O’May, C., Ciobanu, A., Lam, H., and Tufenkji, N. 2012. Tannin derived materials can block 458 swarming motility and enhance biofilm formation in Pseudomonas aeruginosa. Biofouling $459 \quad 28(10):$ 1063-1076.

460 Pompilio, A., Pomponio, S., Crocetta, V., Gherardi, G., Verginelli, F., Fiscarelli, E., et al. 461 2011. Phenotypic and genotypic characterization of Stenotrophomonas maltophilia isolates 462 from patients with cystic fibrosis: genome diversity, biofilm formation, and virulence. BMC 463 Microbiol. 11: 159-175.

464 Rasko, D.A., and Sperandio, V. 2010. Anti-virulence strategies to combat bacteria-mediated 465 disease. Nature Rev. Drug Discov. 9(2): 117-128.

466 Serrano, J., Puupponen-Pimia, R., Dauer, A., Aura, A.M., Saura-Calixto, F. 2009. Tannins: 467 current knowledge of food sources, intake, bioavailability and biological effects. Mol. Nutr. 468 Food Res. 53(2): S310-329. 
469 Sirinavin, S., Techasaensiri, C., Benjaponpitak, S., Pornkul, R., and Vorachit, M. 2005. 470 Invasive Chromobacterium violaceum infection in children: case report and review. Pediatr.

471 Infect. Dis. J. 24(6): 559-561.

472 Taganna, J.C., Quanico, J.P., Perono, R.M., Amor, E.C., and Rivera, W.L. 2011. Tannin-rich 473 fraction from Terminalia catappa inhibits quorum sensing (QS) in Chromobacterium 474 violaceum and the QS-controlled biofilm maturation and Las A staphylolytic activity in 475 Pseudomonas aeruginosa. J. Ethanopharmacol. 134: 865-871.

476 Takahashi, O., Cai, Z., Toda, M., Hara, Y., and Shimamura, T. 1995. Appearance of 477 antibacterial activity of oxacillin against methicillin-resistant Staphylococcus aureus (MRSA) 478 in the presence of catechin. Kansenshogaku Zasshi 69: 1126-1134.

479 Tremblay, J., and Deziel, E. 2008. Improving the reproducibility of Pseudomonas aeruginosa 480 swarming motility assays. J. Basic Microbiol. 48(6): 509-515.

481 Trentin, D.S., Silva, D.B., Amaral, M.W., Zimmer, K.R., Silva, M.V., Lopes, N.P., et al. 482 2013. Tannins possessing bacteriostatic effect impair Pseudomonas aeruginosa adhesion and 483 biofilm formation. PLoS ONE 8(6): e66257 doi:10.1371/journal.pone.0066257.

484 Yahiaoui, Z.R., Zaidi, F. Bessei, A.A. 2008. Influence of gallic and tannic acids on enzymatic 485 activity and growth of Pectobacterium chrysanthemi (Dickeya chrysanthemi bv. 486 Chrysanthemi). African J. Biotechnol. 7(4): 482-486.

487 Yang, C.H., and Li, Y.H. 2011. Chromobacterium violaceum infection: a critical review of an 488 important but neglected infection. J. Chin. Med. Assoc. 74(10): 435-441.

489 Yang, C.H. 2011. Nonpigmented Chromobacterium violaceum bacteremic cellulitis after fish 490 bite. J. Microbiol. Immunol. Infect. 44: 401-405. 
492 Table 1. Growth inhibitory activity of tannins determined by the agar well diffusion method 493

\begin{tabular}{llllllllll}
\hline Tannins $(\boldsymbol{\mu g} / \mathbf{m L})$ & $\mathbf{0}$ & $\mathbf{1 5}$ & $\mathbf{3 0}$ & $\mathbf{6 0}$ & $\mathbf{1 2 5}$ & $\mathbf{2 5 0}$ & $\mathbf{5 0 0}$ & $\mathbf{1 0 0 0}$ & $\mathbf{2 0 0 0}$ \\
\hline Tannic acid (TA) & + & + & + & + & + & - & - & - & - \\
& & & & & & & & & \\
& & & & & & & $(\mathrm{MIC})$ & & \\
Gallic acid (GA) & + & + & + & + & + & + & + & + & -
\end{tabular}

494 Note: "+" corresponds to no inhibition zone, "-" corresponds to inhibition zone with diameter of $495 \geq 1 \mathrm{~mm}$.

496

497

498

499 


\section{Figure captions}

501 Fig. 1. Growth of C. violaceum CV026 in 96 well polystyrene microtiter plates. Growth of the 502 bacterium was estimated at $\mathrm{OD}_{600}$ in presence and absence of (A) tannic acid (TA) and (B) 503 gallic acid (GA). Selected concentrations of TA and GA are shown in the graph.

Fig. 2. Checkerboard assay to determine the effect of (A) tannic acid (TA) and carbenicillin 506 (CAR); (B) gallic acid (GA) and carbenicillin (CAR); (C) tannic acid (TA) and tetracycline 507 (TET) and (D) gallic acid (GA) and tetracycline (TET) on growth of C. violaceum. The black 508 colored wells indicate growth of $C$. violaceum and the white wells represent no growth.

Fig. 3. Effect of tannic acid (TA; A, C) and gallic acid (GA; B, D) in presence of carbenicillin 511 (CAR) and tetracycline (TET) on biofilm formation by C. violaceum CV026. Biofilm 512 absorbance was taken at $\mathrm{OD}_{595}$ and normalized with growth $\left(\mathrm{OD}_{600}\right)$. The bars represent 513 biofilm formation by $C$. violaceum at sub-MIC concentrations of TA $(0-125 \mu \mathrm{g} / \mathrm{mL})$, GA (0$5141000 \mu \mathrm{g} / \mathrm{mL})$ and antibiotics, CAR $(0-6 \mathrm{mg} / \mathrm{mL})$ and TET $(0-7 \mu \mathrm{g} / \mathrm{mL})$. $\dagger$ indicates 515 statistically different values $(P<0.05)$ of antibiotics (CAR or TET) without TA or GA when 516 compared with control (values without CAR or TET) and * indicates statistically different 517 values $(P<0.05)$ of TA or GA when compared with control (values without TA or GA) at 518 different concentrations of antibiotics (CAR or TET).

520 Fig. 4. Effect of (A) tannic acid, TA and (B) gallic acid, GA on swimming motility of $C$. 521 violaceum $\mathrm{CV} 026 .{ }^{*}$ indicates statistically different values $(P<0.05)$ when compared to control 522 without tannins. 
524 Fig. 5. Molecular structures of tannin compounds used in this study.

525 

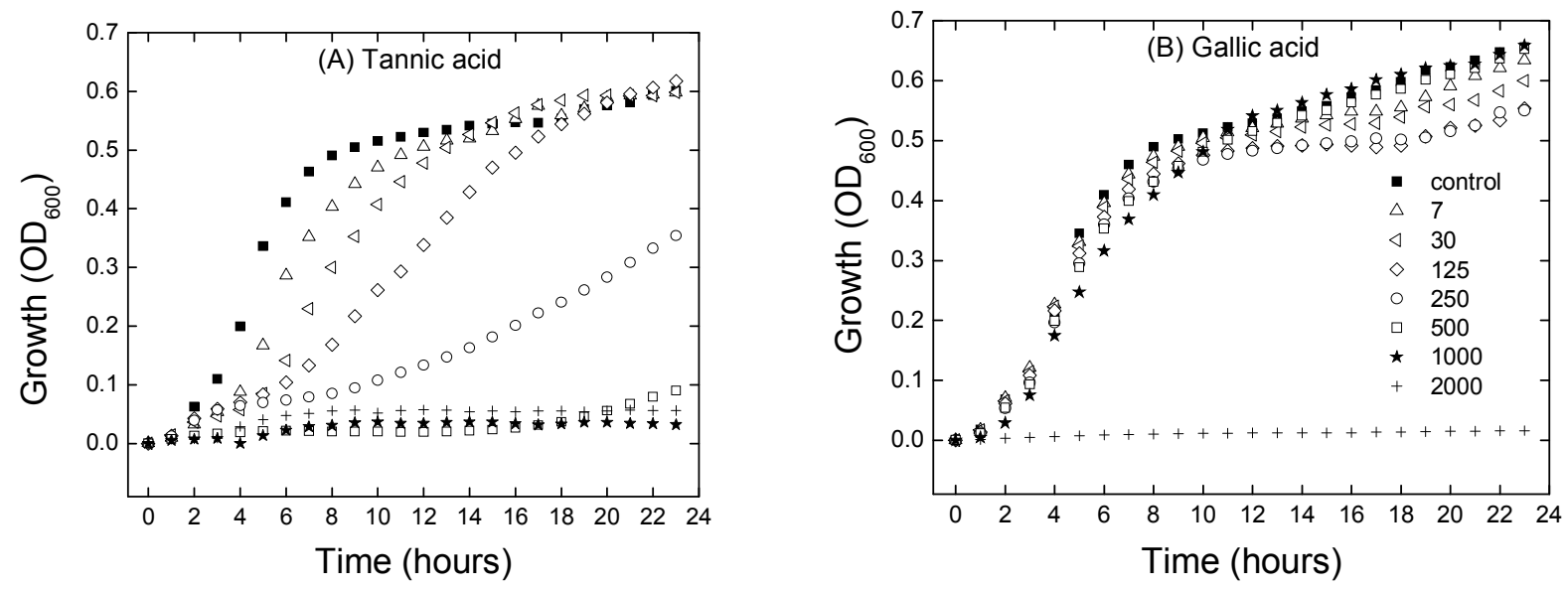

Fig. 1. Growth of $C$. violaceum CV026 in 96 well polystyrene microtiter plates. Growth of the bacterium was estimated at $\mathrm{OD}_{600}$ in presence and absence of (A) tannic acid (TA) and (B) gallic acid (GA). Selected concentrations of TA and GA are shown in the graph. 


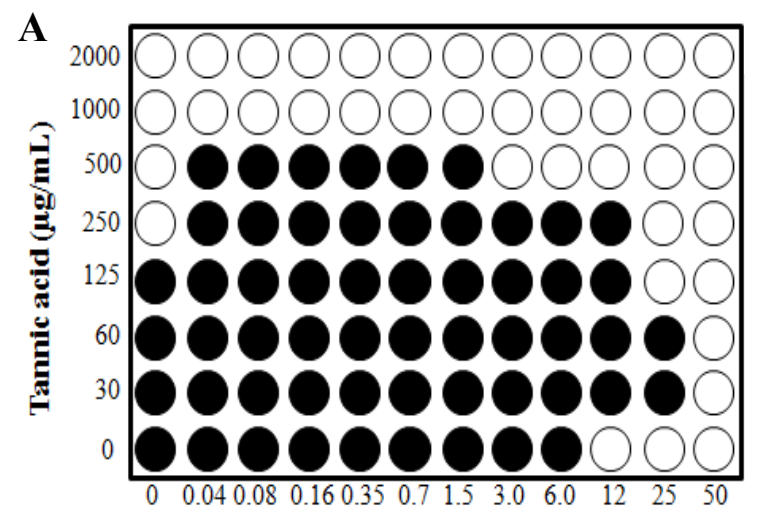

Carbenicillin $(\mathrm{mg} / \mathrm{mL})$

\section{C}

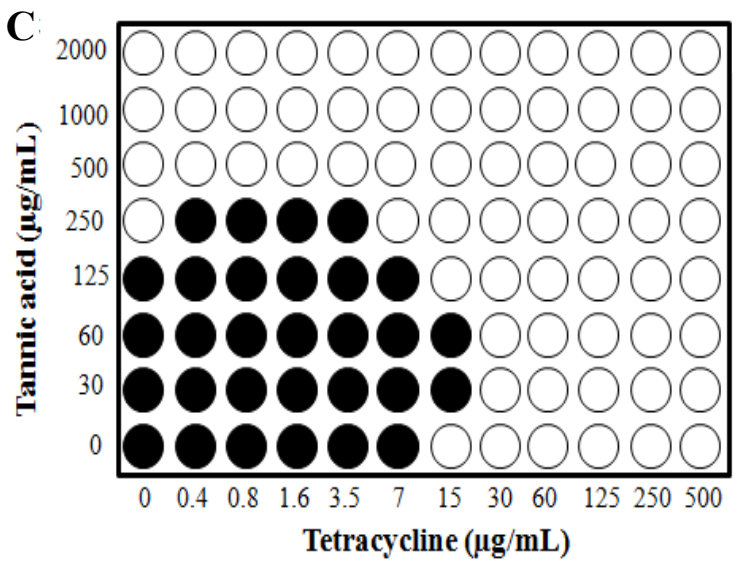

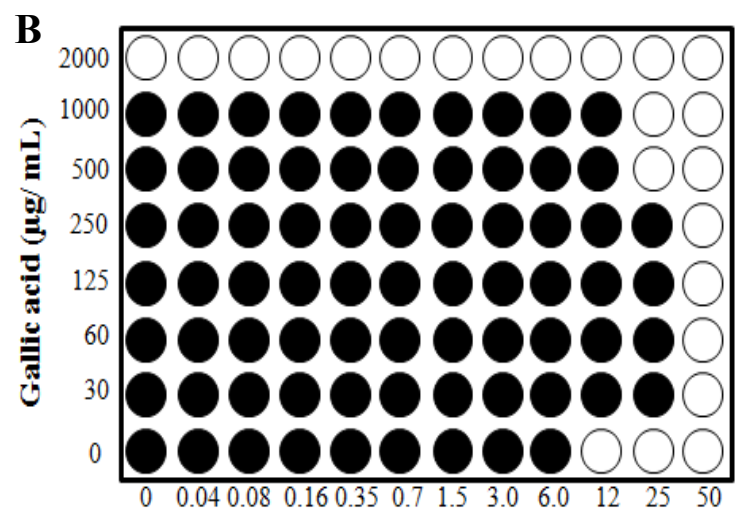

Carbenicillin (mg/mL)

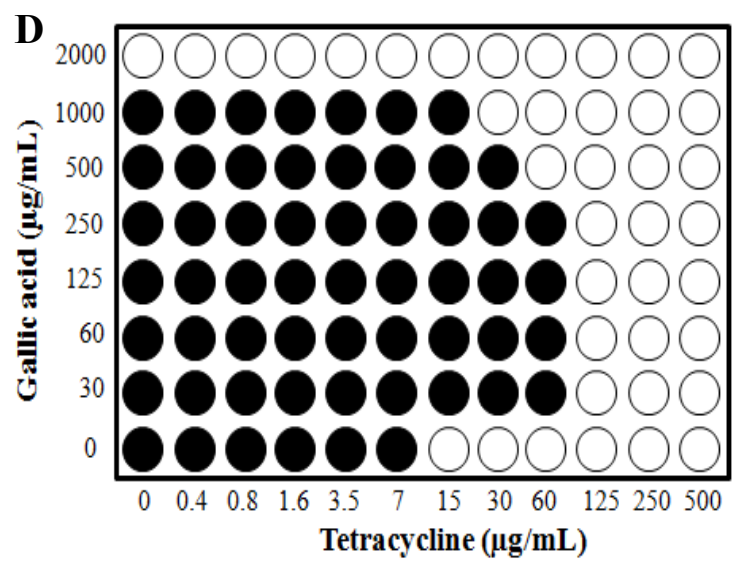

Fig. 2. Checkerboard assay to determine the effect of (A) tannic acid (TA) and carbenicillin (CAR); (B) gallic acid (GA) and carbenicillin (CAR); (C) tannic acid (TA) and tetracycline (TET) and (D) gallic acid (GA) and tetracycline (TET) on growth of $C$. violaceum. The black colored wells indicate growth of $C$. violaceum and the white wells represent no growth. 

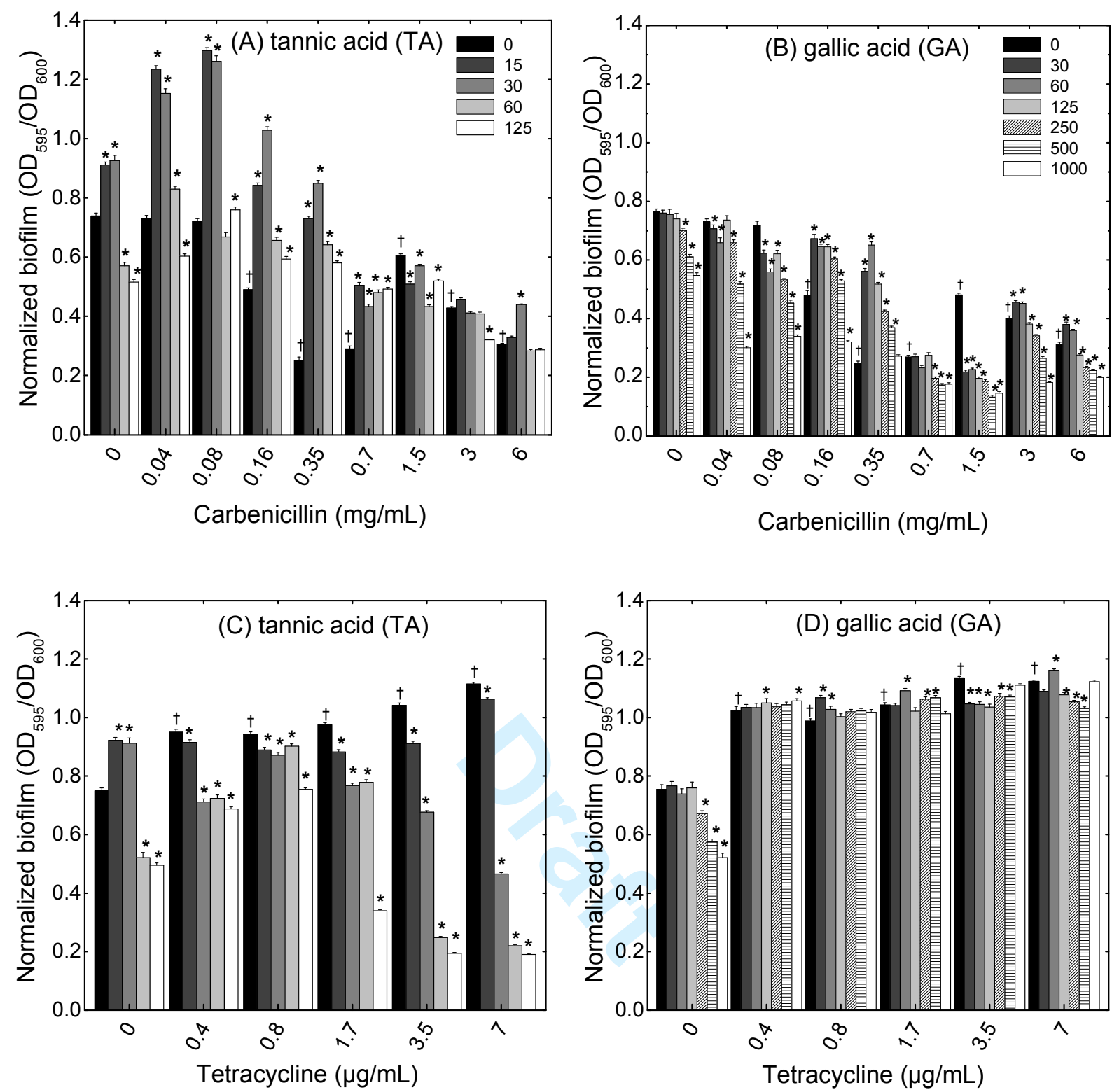

Fig. 3. Effect of tannic acid (TA; A, C) and gallic acid (GA; B, D) in presence of carbenicillin (CAR) and tetracycline (TET) on biofilm formation by $C$. violaceum CV026. Biofilm absorbance was taken at $\mathrm{OD}_{595}$ and normalized with growth $\left(\mathrm{OD}_{600}\right)$. The bars represent biofilm formation by $C$. violaceum at sub-MIC concentrations of TA $(0-125 \mu \mathrm{g} / \mathrm{mL})$, GA (0$1000 \mu \mathrm{g} / \mathrm{mL})$ and antibiotics, CAR $(0-6 \mathrm{mg} / \mathrm{mL})$ and TET $(0-7 \mu \mathrm{g} / \mathrm{mL}) .{ }^{\dagger}$ indicates statistically different values $(P<0.05)$ of antibiotics $($ CAR or TET) without TA or GA when compared with control (values without CAR or TET) and ${ }^{*}$ indicates statistically different values $(P<0.05)$ of TA or GA when compared with control (values without TA or GA) at different concentrations of antibiotics (CAR or TET). 

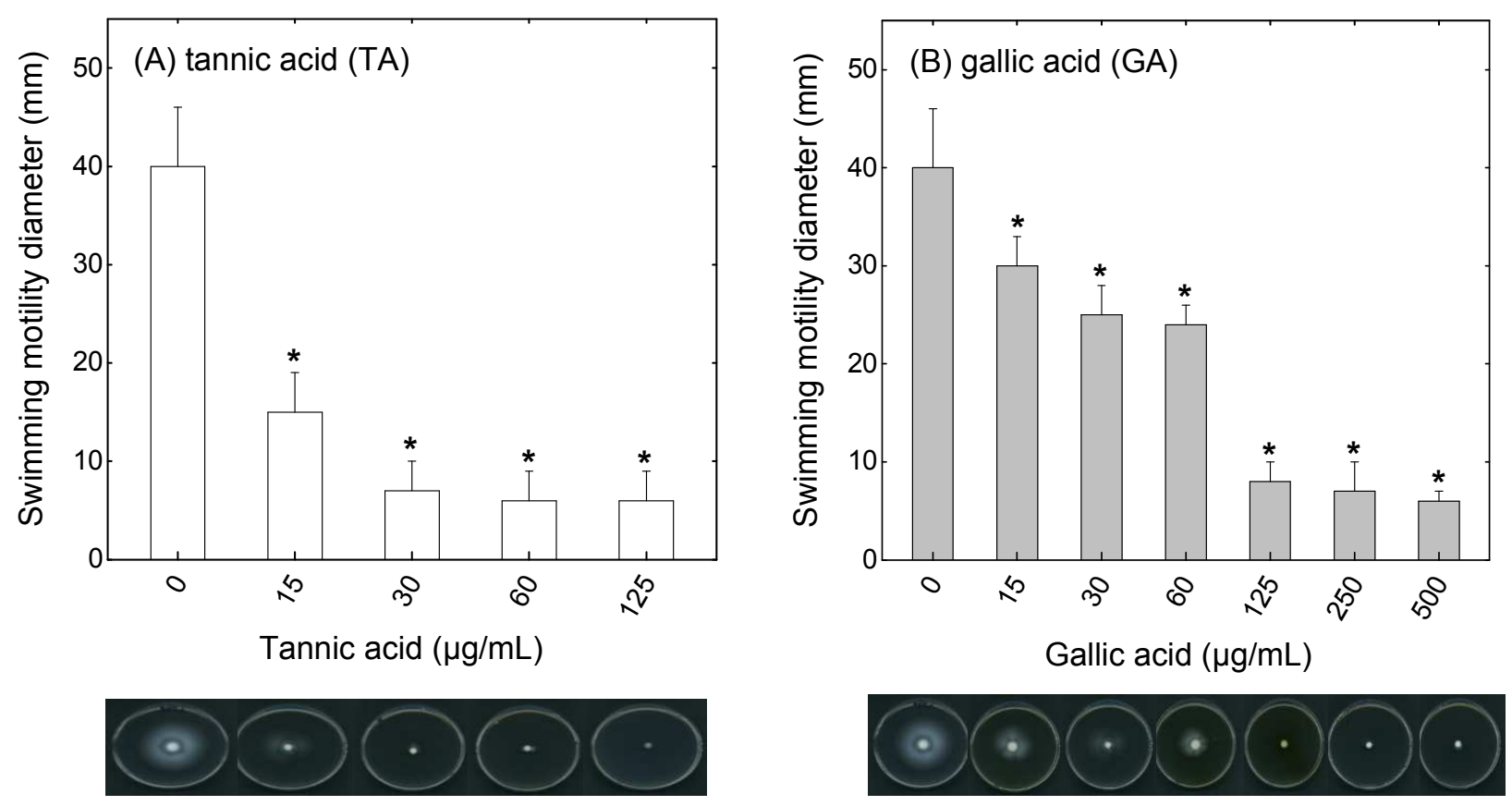

Fig. 4. Effect of (A) tannic acid, TA and (B) gallic acid, GA on swimming motility of $C$. violaceum $\mathrm{CV} 026 .{ }^{*}$ indicates statistically different values $(P<0.05)$ when compared to control without tannins. 


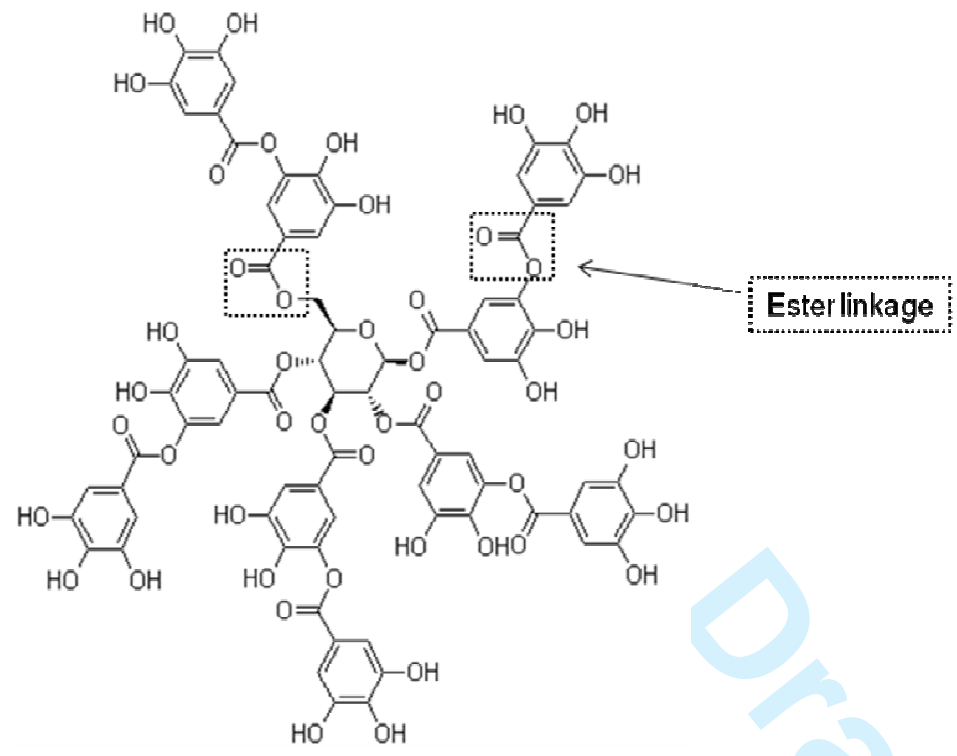

Tannic acid (TA)

(MW: 1701.2)<smiles>O=C(O)c1cc(O)c(O)c(O)c1</smiles>

Gallic acid (GA)

(MW: 170.12)

Fig. 5. Molecular structures of tannin compounds used in this study. 